\title{
Keratoglobus after Long Standing Keratoconus - A Rare Case
}

\author{
Pravin K. Tidke1, Neha Bajpayee ${ }^{2}$ \\ 1, 2 Department of Ophthalmology, Jawaharlal Nehru Medical College, Datta Meghe Institute of Medical Sciences \\ (Deemed to Be University), Sawangi (Meghe), Wardha, Maharashtra, India.
}

\section{INTRODUCTION}

Keratoglobus can be congenital or acquired. Congenital form always is associated with connective tissue disorder like Ehlers Danlos syndrome type 4 and blue sclera syndrome. Acquired form occurs in later stages of life and can be associated with keratoconjunctivitis, pellucid marginal degeneration, and is a severe form of keratoconus. Keratoglobus is a minimally progressive disorder and when presents with keratoconus, corneal thickness is compromised, thus, proper evaluation of cornea is needed. Since prevalence of keratoglobus in Central India is $2.3 \%$, and association of this with keratoconus makes this case more unusual to study and report.

Keratoglobus is non-inflammatory and ectatic disorder of cornea which causes corneal thinning from limbus to limbus and leads to globular protrusion of cornea. ${ }^{1}$ In past keratoglobus was misdiagnosed as megalocornea or congenital glaucoma. ${ }^{2}$ The aetiology remains unknown but there is similarities in aetiology with common inflammatory ectatic conditions like keratoconus. ${ }^{2,3}$ Keratoglobus generally are congenital disorders but congenital forms are often bilateral and associated with connective tissue disorder like blue sclera. ${ }^{4,5}$ Ehlers-Danlos syndrome ${ }^{6}$ is autosomal recessive. $^{7}$ Acquired form can occur in later stages of life, secondary to keratoconus, 8,9 pellucid marginal degeneration, 10 keratoconjunctivitis, chronic marginal blepheritis ${ }^{11}$ where corneal ectasia is due to constant eye rubbing.

With globular protrusion of cornea, there is corneal thinning from limbus to limbus. Thickness reduces to one third of normal corneal thickness. If there is no scarring, acute hydrops patient often presents with clear cornea. ${ }^{2}$ Main cause of poor vision in such patients is high myopia and irregular astigmatism which is due to protrusion. Hence, correction of refractive error is difficult. Since there is marked corneal thinning, even small trauma can lead to corneal perforation. $2,4,8$ In such cases corneal repairing and resuturing becomes a difficult task due to instability of cornea and reduced thickness in keratoglobus.
Corresponding Author:

Dr. Neha Bajpayee,

S-16 Shalintai PG Girls Hostel,

Datta Meghe Institute of Medical

Sciences (Deemed to Be University),

Sawangi (Meghe), Wardha,

Maharashtra, India.

E-mail: nehabajpayee1601@gmail.com

DOI: $10.14260 / j e m d s / 2021 / 87$

How to Cite This Article:

Tidke PK, Bajpayee N. Keratoglobus after long standing keratoconus- a rare case. J Evolution Med Dent Sci 2021;10(06):394398, DOI: $10.14260 / \mathrm{jemds} / 2021 / 87$

Submission 09-09-2020,

Peer Review 01-12-2020,

Acceptance 07-12-2020,

Published 08-02-2021.

Copyright (C) 2021 Pravin K. Tidke et al. This is an open access article distributed under Creative Commons Attribution License [Attribution 4.0 International (CC BY 4.0)] 
Diagnosis of these cases is purely clinical. To differentiate it from other ectatic conditions two modalities that are used are ultrasound pachymetry, specular microscopy for determining reduced corneal thickness and corneal topography for measuring diffuse corneal thinning. ${ }^{12,13}$ Flattening of 'arching' of the bow tie configuration due to peripheral arc of increases power or steepening is seen in topography.2,13 To rule out connective tissue disorder systemic investigations are required. Signs like blue sclera, joint hypermobility, skeletal abnormalities, hearing abnormalities, high arch palate should be properly examined. Histopathology of cornea in keratoglobus of congenital form shows frequent disruptions and absence of Bowman's membrane, disorganisation or breaks in Descemet's membrane, and stromal thinning. ${ }^{14}$ Acquired type has normal Bowman's layer and there is focal breaks with superficial stromal ectasia secondary to other corneal pathology. ${ }^{7}$ In keratoglobus corneal thinning is diffuse and has globular protrusion, ${ }^{9}$ in keratoconus thinning is mainly at apex as protrusion is conical in shape, in pellucid marginal degeneration thinning is at inferior aspect of cornea mainly at 4 - 8'o clock and thinning is superior to that.

Treatment is challenging in keratoglobus. Conservative treatment like correcting refractive errors for high myopia and irregular astigmatism is given. Conventionally, treatment includes corneal transplants, that is, keratoplasties.

\section{PRESENTATION OF CASE}

A 45-year-old female patient presented to ophthalmology outpatient department (OPD) for eye examination with complaint of diminution of vision in right eye in the past 8 months and left eye since 12 months. Diminution of vision was gradual in onset and painless and she was having difficulty to see far off objects more than near-by objects. Patient had no history of spectacle usage, redness in eyes, night blindness, peripheral vision loss, headache, coloured halos, or floaters. Patient had no systemic complaints like joint laxity, pain, weakness in joint.

On examination: right eye - visual acuity - 6 / 60 and with pinhole, improvement was up to $6 / 24$. Near vision when given to the patient she could only read N12 of Snellen's near vision chart with addition of $+1.5 \mathrm{D}$ according to her age, auto refractometer could not focus due to irregularity of cornea, patient was not accepting spectacles. Eyelid, conjunctiva, iris, anterior chamber was normal

Cornea showed positive Munson's sign with conical shape (Figure 2). In lens there was no abnormality detected.

Intraocular pressure was $12 \mathrm{~mm}$ of $\mathrm{Hg}$ measured by noncontact tonometer. Extraocular movements were free and full in all directions of gaze.

Corneal topography was performed, and it showed vertical axis was $56.28 \pm 7.78 \mathrm{D}$ and horizontal axis was 47.48 $\pm 7.78 \mathrm{D}$.

In this image axial maps and tangential maps (Figure 1) are showing inferior steepening along with paracentral steepening and there is decentering of the corneal apex from visual axis. Elevation map is showing elevation superiorly. This indicates patient has conical corneal curvature, hence, suggesting of keratoconus, leading to keratoglobus.
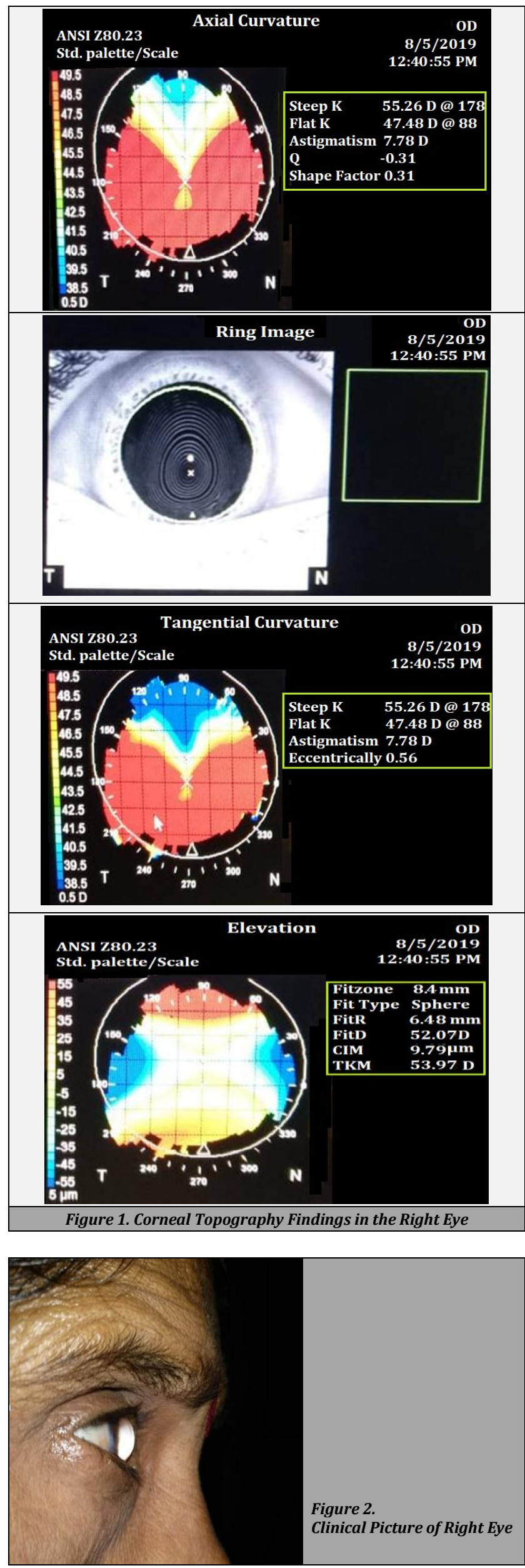


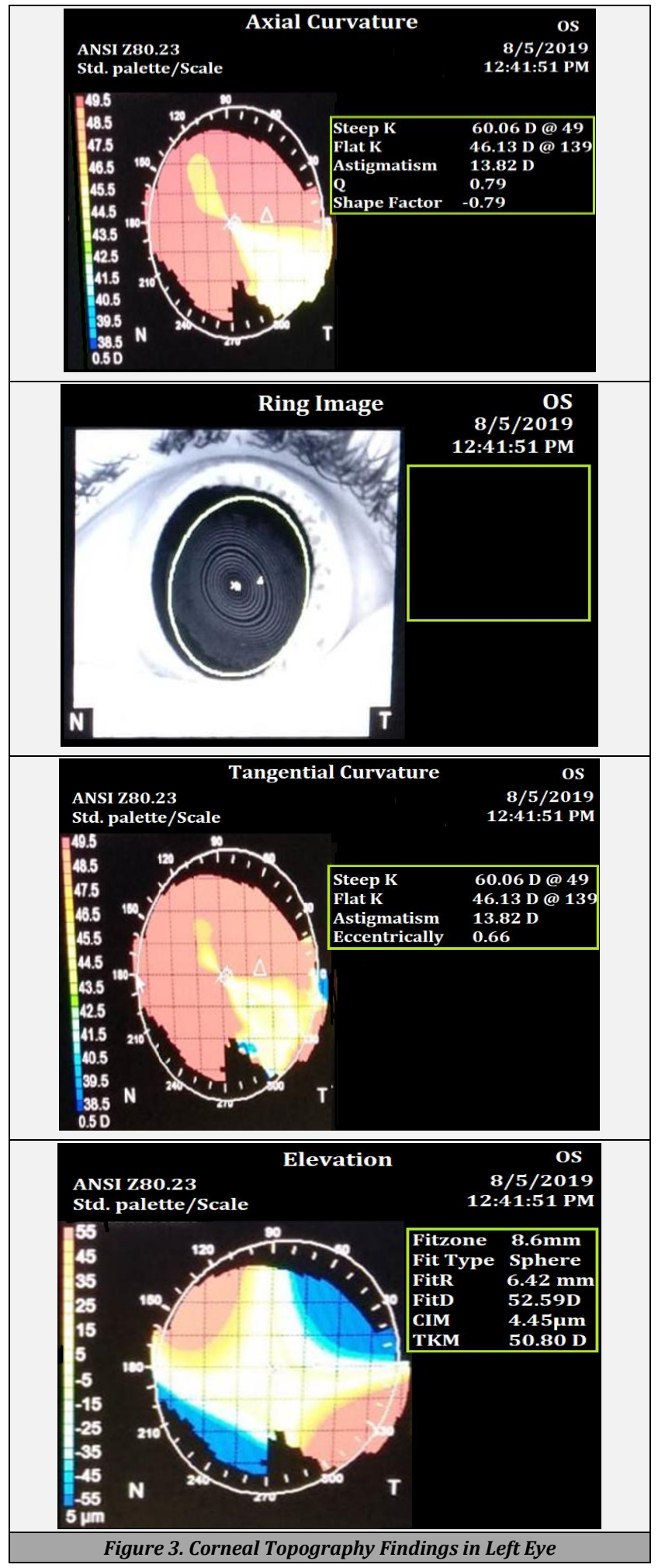

Specular microscopy of right eye suggested to have central corneal thickness of $509 \mu \mathrm{m}$, cell density was 1942 cells / $\mathrm{mm}^{2}$, coefficient of variation was $33 \%$ and hexagonality was $49 \%$. This data suggested that there is marked thinning all over the cornea with endothelial decompensation.

Fundus examination: media was clear, disc was normal, blood vessels were normal and foveal reflex was bright.

On examination of left eye - visual acuity - 6 / 60 and with pinhole improvement was up to $6 / 36$, near vision when given to the patient she could only read N12 of Snellen's near vision chart with addition of $+1.5 \mathrm{D}$ according to her age, auto refractometer could not focus due to irregularity of cornea, patient was not accepting spectacles. Eyelid, conjunctiva, iris, anterior chamber was normal

Cornea showed positive Munson's sign with conical shape (Figure 4). There was no abnormality detected in lens.

Intraocular pressure was $14 \mathrm{~mm}$ of $\mathrm{Hg}$, measured by noncontact tonometer. Extraocular movements were free and full in all directions of gaze.

Corneal topography was performed, and it showed vertical axis was $60.05 \pm 13.82 \mathrm{D}$ and horizontal axis was $48.13 \pm 13.82 \mathrm{D}$.

In this image axial maps and tangential maps (Figure 3) are showing superior steepening along with paracentral steepening and mild inferior steeping. There is decentering of the corneal apex from visual axis. Elevation map is showing elevation supero-temporaly and infero-nasally. This suggests that left eye surface of the cornea is more irregular than right eye with higher astigmatism.

Specular microscopy of left eye suggested to have central corneal thickness of $480 \mu \mathrm{m}$, cell density was 1742 cells / $\mathrm{mm}^{2}$, coefficient of variation was $29 \%$ and hexagonality was $38 \%$. Hence, this shows left eye has major corneal endothelial decompensation than right eye.
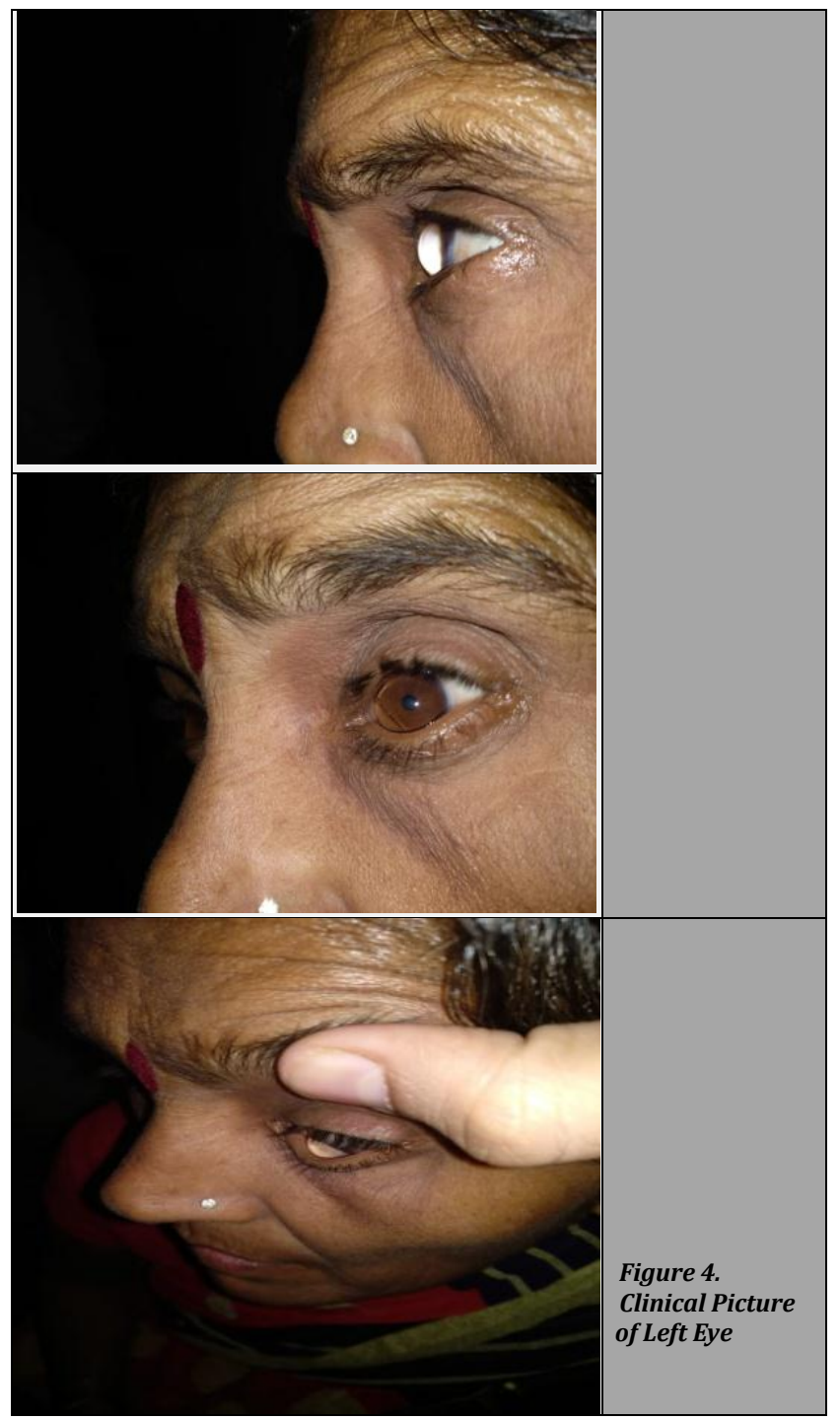

Fundus examination: Media was clear, disc was normal, blood vessels were normal and foveal reflex was bright. On 
slit lamp biomicroscopy - "oil drop reflex" was seen in both eyes.
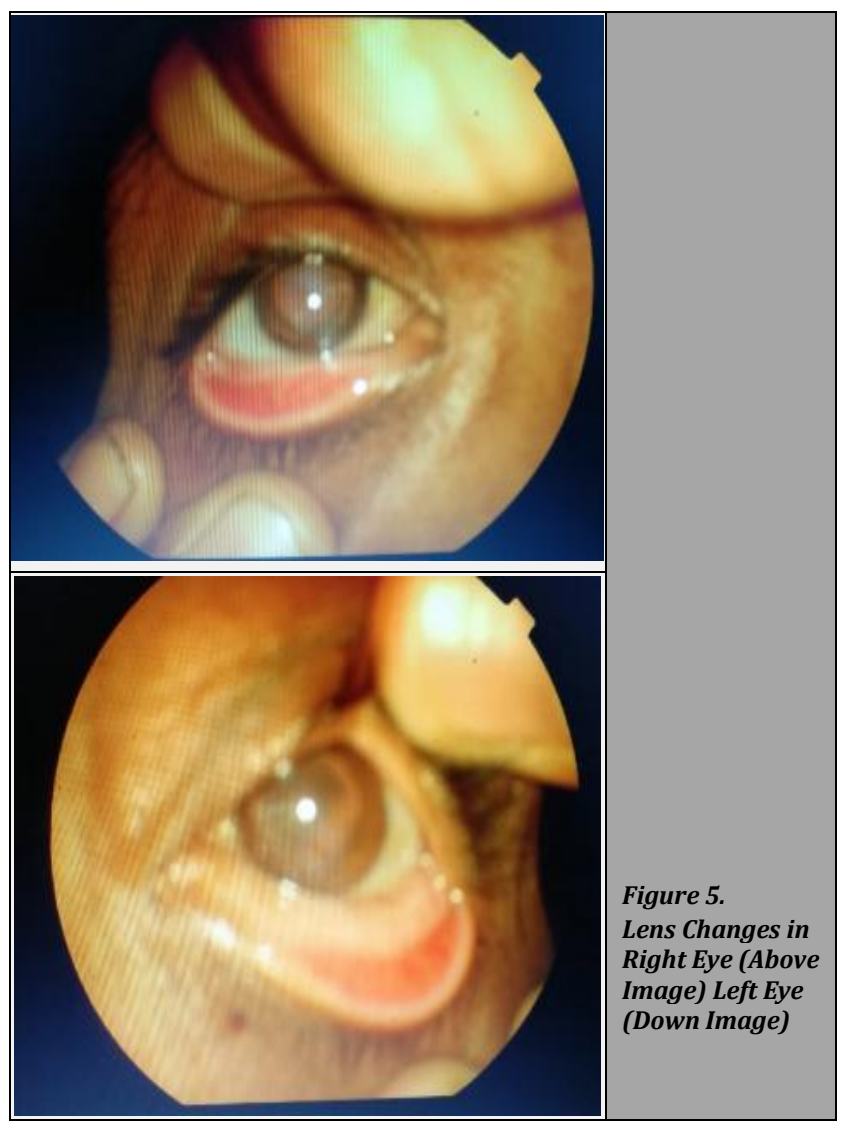

Laboratory investigation of this patient was done, to rule out any connective tissue disorder such as complete blood count, erythrocyte sedimentation rate (ESR), c-reactive protein, urine microscopic analysis, serum creatinine, rheumatoid factor, antinuclear antibody titers. All the investigations were within normal limit.

\section{DISCUSSION OF MANAGEMENT}

As, patient had higher amount of astigmatism in both eyes, best corrected refraction through subjective method was provided which led to improvement till 6 / 18 on application of (- 4D cylindrical) in right eye and 6 / 24 after application of (- 6D cylindrical) in left eye. Patient was not advised contact lenses due to marked corneal thinning and higher chances of perforation. Surgical option of penetrating keratoplasty was given to the patient and guarded visual prognosis was explained, but patient refused to give consent for surgery and thus, patient was discharged with best corrected refraction.

\section{DISCUSSION}

Keratoglobus being the non-inflammatory and corneal thinning disorder leads to globular ectasia (protrusion) and scarring of cornea. Often perforation may follow after trivial trauma. Mainly, bilateral keratoglobus is associated with connective tissue disorders, but in this case keratoglobus was a presentation of long-standing keratoconus.
Diagnosis of such cases are mostly made clinically, but for knowing actual curvature of cornea, performing corneal topography plays a major role. There are various maps in topography which help us to examine cornea from different axis. Axial maps and tangential maps are two categories which are used for screening of curvature of cornea thus, helps in diagnosing a pathology like keratoconus. Axial maps are less sensitive for corneal curvature measuring hence, used only for screening.

As keratoconus has a prevalence of $2.3 \%+/-0.2 \%$ among Indians, aged 30 years and above, and living in the rural regions of Central India; this case becomes more unique as it is associated with acquired keratoglobus.

Patients generally have clear cornea and usually present with gradual diminution of vision in case of keratoglobus. Keratoglobus is a minimally progressive disorder but in this case as this presents with keratoconus it becomes a matter of concern as corneal thinning is seen majorly at inferior and paracentral aspect covering the visual axis in right eye and superiorly and supero-temporally and inferio-nasally paracentrally in left eye. There was reduced corneal thickness and decompensated endothelium in both eyes when parameters were taken with specular microscopy.

Since, in this case patient has high myopia and high irregular astigmatism, contact lenses were not advised as even a small trauma while applying or removing contact lens on such thinned cornea may increase chances of perforation and, acute hydrops. Thus, giving best possible refractive correction was the choice or treatment that was preferred. Other modality of treatment is surgical which includes penetrating keratoplasty limbus to limbus type, but since in this case corneal curvature is highly irregular with irregular astigmatism, chances of graft rejection, and disparity between peripheral graft host thicknesses makes it difficult for wound closure.

Other treatment measures that can be used are small diameter rigid gas permeable (RGP) lenses, reverse geometry hydrogel lenses, as well as large diameter inverse geometry RGP lenses. These are customised fitting lenses on patient-topatient basis which have been described for corneal ectasias, and sclera lenses. ${ }^{15,16}$ Surgical newer techniques include lamellar keratoplasty especially epikeratoplasty. It has higher chances of infection but has proven to have better visual outcomes than conventional penetrating keratoplasty. ${ }^{17}$ Other options like tuck-in lamellar keratoplasty has technical difficulties and causes interface opacities. ${ }^{18}$ Pentacam based deep anterior lamellar keratoplasty is technically demanding. ${ }^{19}$ Corneoscleral rim provides tectonic stability but is a temporary measure. ${ }^{20}$

Among these surgical procedures, there is no standard surgery for keratoglobus, due to rarity of the case and limited follow up of newer surgical procedures. ${ }^{18-21}$ Therefore, visual rehabilitation and maintaining structural integrity of cornea still remains a challenge.22-24 Post-operative follow-up evaluation is also very important. ${ }^{25-26}$

Financial or other competing interests: None.

Disclosure forms provided by the authors are available with the full text of this article at jemds.com. 


\section{REFERENCES}

[1] Rathi VM, Murthy SI, Bagga B, et al. Keratoglobus: an experience at a tertiary eye care center in India. Indian J Ophthalmol 2015;63(3):233-8.

[2] Wallang BS, Das S. Keratoglobus. Eye (Lond) 2013;27(9):1004-12.

[3] Verrey F. Keratoglobeaigu. Ophthalmologica 1947;114(4-5):284-8.

[4] Gregoratos ND, Bartsocas CS, Papas K. Blue sclerae with keratoglobus and brittle cornea. Br J Ophthalmol 1971;55(6):424-6.

[5] Biglan AW, Brown SI, Johnson BL. Keratoglobus and blue sclera. Am J Ophthalmol 1977;83(2):225-33.

[6] Cameron JA. Corneal abnormalities in Ehlers-Danlos syndrome type VI. Cornea 1993;12(1):54-9.

[7] Pouliquen Y, Dhermy P, Espinasse MA, et al. Keratoglobus. J Fr Ophthalmol 1985;8(1):43-54.

[8] Baillif S, Garweg JG, Grange JD, et al. Keratoglobus: review of the literature. J Fr Ophthalmol 2005;28(10):1145-9.

[9] Cavara V. Keratoglobus and keratoconus. A contribution to the nosological interpretation of keratoglobus. $\mathrm{Br} \mathrm{J}$ Ophthalmol 1950;34(10):621-6.

[10] Rumelt S, Rehany U. Surgically induced keratoglobus in pellucid marginal degeneration. Eye (Lond) 1998;12(Pt 1):156-8.

[11] James AC. Keratoglobus. Cornea 1993;12(2):124-30.

[12] Karabatsas $\mathrm{CH}$, Cook SD. Topographic analysis in pellucid marginal corneal degeneration and keratoglobus. Eye (Lond) 1996;10(Pt 4):451-5.

[13] Lee BW, Jurkunas UV, Harissi-Dagher M, et al. Ectatic disorders associated with a claw-shaped pattern on corneal topography. Am J Ophthalmol 2007;144(1):1546.

[14] Meghpara B, Nakamura H, Vemuganti GK, et al. Histopathologic and immunohistochemical studies of keratoglobus. Arch Ophthalmol 2009;127(8):1029-35.

[15] Pullum KW, Buckley RJ. A study of 530 patients referred for rigid gas permeable scleral contact lens assessment. Cornea 1997;16(6):612-22.
[16] Foss AJ, Trodd TC, Dart JK. Current indications for scleral contact lenses. CLAO J 1994;20(2):115-8.

[17] Javadi MA, Kanavi MR, Ahmadi M, et al. Outcomes of epikeratoplasty for advanced keratoglobus. Cornea 2007;26(2):154-7.

[18] Vajpayee RB, Bhartiya P, Sharma N. Central lamellar keratoplasty with peripheral intralamellar tuck: a new surgical technique for keratoglobus. Cornea 2002;21(7):657-60.

[19] Riss S, Heindl LM, Bachmann B0, et al. Pentacam-based big bubble deep anterior lamellar keratoplasty in patients with keratoconus. Cornea 2012;31(6):627-32.

[20] Kanellopoulos AJ, Pe LH. An alternative surgical procedure for the management of keratoglobus. Cornea 2005;24(8):1024-6.

[21] Jonas JB, Nangia V, Matin A, et al. Prevalence and associations of keratoconus in rural Maharashtra in central India: the central India eye and medical study. Am J Ophthalmol 2009;148(5):760-5.

[22] Pareek A, Khandekar A, Acharya S, et al. Correlation between nephropathy and ophthalmic complications in cases of sickle cell anemia: an entangled association. Indian Journal of Medical Specialities 2019;10(2):72-5.

[23] Prasad M, Daigavane S, Kalode V. Visual outcome after cataract surgery in rural hospital of Wardha district: a prospective study. Journal of Clinical and Diagnostic Research 2020;14(2):4-6.

[24] Daigavane S, Patkar P. To compare the changes in the corneal endothelium post phacoemulsification surgery with balanced salt solution vs. viscoelastic device. Journal of Clinical and Diagnostic Research 2019;13(12):1-4

[25] Patkar P, Sune P. Evaluation of tear film functions preoperatively and postoperatively in cases with pterygium: a case control study. Journal of Clinical and Diagnostic Research 2020;14(1):NC10-3.

[26] Mukherji R, Sune S. Contrast sensitivity and visual acuity before and after Nd: YAG capsulotomy in patients with posterior capsular opacification: a prospective study. Journal of Clinical and Diagnostic Research 2020;14(3):5-8. 